\title{
Cytogenetic differences in blood and cancer tissue samples of the
}

\section{same patient group}

\section{Osman Demirhan ${ }^{1 *}$, Deniz Taştemir Korkmaz ${ }^{2}$ and Nesrin Çetinel ${ }^{1}$}

1 Department of Medical Biology, Faculty of Medicine, Çukurova University, 01330 Balcal1-Adana, Turkey.

2 Department of Medical Biology, Faculty of Medicine, Adıyaman University, Adiyaman, Turkey.

*Corresponding Author: Prof. Dr. Osman Demirhan, Department of Medical Biology, Faculty of Medicine, Çukurova University, and 01330

Balcal1-Adana, Turkey, GMS: 05060229765, e-mail: osdemir@cu.edu.tr, odemirhan42@gmail.com.

Received date: July 23, 2020; Accepted date: July 31, 2020; Published date: Augusł 07, 2020

Citation: Osman Demirhan, Deniz Taştemir Korkmaz and Nesrin Çetinel,;Cytogenetic differences in blood and cancer tissue samples of the same patient group; J Cancer Research and Cellular Therapeutics;3(1);DOI: 10.31579/2640-1053/070

Copyright: () 2020, Osman Demirhan: This is an open access article distributed under the Creative Commons Attribution License, which permits unrestricted use, distribution, and reproduction in any medium, provided the original work is properly cited.

\begin{abstract}
Breast cancer (BC) is the most prevalent malignant disease in females worldwide. Genomic instability in tumor tissue has been associated with tumor progression. These genetic changes may take a variety of forms, including numerical and structural chromosomal abnormalities (CAs), epigenetic changes, and gene expression alterations. Many tumor tissues are made up of genetically different cell populations, and the study of the causes and consequences of this heterogeneity play a central role in cancer research. In this study, CAs in blood and cancer tissues of patients with sporadic BC were examined. Our findings shows that the increase in numerical sex aneuploidy in BC tissues is significantly higher than in blood tissue. These aneuploidy increases in cancer tissues seem to be compatible with the development and increase of cancer, and can play a role in the pathogenesis of cancers. These changes are consistent with early and long-standing exposure to carcinogens, especially estrogens. These findings should clarify our understanding of breast carcinogenesis in breast tissues and promote development of improved methods for risk assessment and $\mathrm{BC}$ prevention in women.
\end{abstract}

Keywords: breast cancer, chromosomal aberrations, anöploidy, blood tissue, cancer tissue

\section{Introduction}

Breast cancer is a major global health problem and the incidence of the disease continues to increase steadily. Sporadic BC develops through the accumulation of genetic abnormalities in normal breast tissue, resulting from exposure to estrogens and other carcinogens beginning at adolescence and continuing throughout life. Technical advances in the field of cancer cytogenetics have greatly enhanced the detection ability of CAs, and have facilitated the research and diagnostic potential of chromosomal studies in neoplasms. Cytogenetic and molecular genetic date are of paramount importance in the diagnosis, prognosis, and risk stratification of patients with malignant diseases. Diagnosis of diseasespecific genetic abnormalities can provide the information necessary to assist the oncologist in diagnosing. The DNA in every cell is essentially identical to every other cell. Karyotyping of cancer cells is considered the gold standard method considering that abnormalities in the genome of a single cell are similar to all body cells. Therefore, blood and tissue cells are expected to be genetically matched. Technical advances in the field of cancer cytogenetics, chromosome analysis of a single cell is still the easiest way to delineate and understand the relationship between clonal evolution and disease progression of cancer cells. Therefore, the vast majority of genetic samples used in large-scale studies come in the form of blood. But, there are questions about genetic research based only on blood samples. Because, it is suggested that the genetic damage in blood and tissue cells is different in the same person. CAs are one of the hallmarks of neoplastic cells in general, and the persistent presence of chromosome instability has been demonstrated in many human cancers. Breast carcinomas are among the most heterogeneous human neoplasms. There is more than one genetic damage in the development of malignant BC. These aberrations range from single nucleotide point mutations to structural CAs and gross changes in chromosome copy number. BC progression is characterized by the accumulation of numeric changes on many chromosomes, particularly frequent increases in the copy numbers of chromosomes. Sex chromosome aneuploidy increase was reported to be very high in sarcoma tumor tissue (specifically, polysomies) and in blood tissue, respectively $(\mathrm{p}<0.005)$ [1]. The majority of malignant tumors consists of cells with an aneuploid karyotype. Aneuploidy is a defining feature of cancers with an impact on the genesis, progression and prognosis of these malignancies. The relationship of tumorigenesis and aneuploidy remains enigmatic despite growing scientific interest. Thus, aneuploidy takes numerous variable features and is remarkably demanding to study. It is therefore reasonable to assume that aneuploidy plays a crucial role during carcinogenesis. There have also been casereports suggesting the possibility of increased risks of many other cancers. Therefore, we examined BC clonal sex chromosomal heterogeneity in cancer breast tissue and blood samples by cytogenetic and fluorescence in situ hybridization (FISH) methods.

\section{Materials and methods}

Technical advances in the field of cancer cytogenetics have facilitated the research and diagnostic potential of chromosomal studies 
in neoplasms. In two studies, in two different patient groups, blood tissues of 51 patients and cancer tissue of 49 breast cancer patients were reffered to Department of Medical Biology and Genetics, Faculty of Medicine, Çukurova University for chromosomal analysis. This study was approved by the Ethics Committee of Medical Faculty, Çukurova University. The blood samples were 51 women with $\mathrm{BC}$, to evaluate these women at retrospectively. The average age of the patientswas 44.6 years (between 10 and 80 years). Metaphase chromosome preparations from peripheral blood tissues were made by the standard cytogenetic protocols. In the other group, 49 fresh cancer tissues were analyzed by FISH method. Cancer tissue biopsies were taken by the Department of General Surgery of Çukurova University Faculty of Medicine with mastectomy. Also, the blood tissues of 32 healty age and sex-matched controls were made by the standard cytogenetic protocols (the mean age 29,44 $\pm 5,003$, ranged 21 38). For the purpose of the study, we used CEP X Spectrum Orange DNA Probe Kit (Abbott). FISH was applied according to the method mentioned by Taştemir et al. (2011) [2]. An average of 500 interphase cells was analyzed using a BX51 Olympus fluorescence microscope equipped with Cytovision Probe Software (Applied Imaging, Santa Clara, CA). Monosomic and polysomic cells for $\mathrm{X}$ chromosome positive cells were recorded. Also, cytogenetic analysis of blood samples were conducted by Giemsa-banding and reported according to the current international standard nomenclature (ISCN, 2009) [3].

\section{Results}

We evaluated cytogenetic abnormalities in blood and cancer tissues of total 100 histopathologically confirmed BC patients, and 32 females as control. Blood samples of 51 patients was conducted by Giemsa-banding. The karyotype results were abnormal in $31,4 \%$ of all patients. Structural and numerical CAs were observed in 27,5\% and 3,9\% of patients, respectively. Specifically, the deletions and fragilities were the most common karyotypes $(7,8 \%)$. Translocations were detected in $2 \%$ and the frequency of other CAs was $5,9 \%$ of the patients. Aneuploidies detected in $3,9 \%$ of the patients. The $7 \mathrm{p} 11,7 \mathrm{q} 32,9 \mathrm{p} 32,11 \mathrm{p} 15,11 \mathrm{q} 12$, $11 \mathrm{q} 23,21 \mathrm{q} 11,21 \mathrm{q} 21,15 \mathrm{p} 11$ and $17 \mathrm{q} 23$ regions were the most common chromosome damage observed in patients. These regions can be play an important role in the development of $\mathrm{BC}$ and requires further molecular analysis as a potential candidate region for the isolation of genes related to carcinogenesis. In another patient group, we examined only sex chromosome numerical aberrations in 49 cancer tissues by FISH. There were many $\mathrm{X}$ chromosome losses and gains in tumor tissue cells. At least one $\mathrm{X}$ chromosome loss (monosomy)/gain (polysomy) was recorded in $35.68 \%$ of the cells examined. $X$ chromosome polysomies were categorized as $3 \mathrm{X}, 4 \mathrm{X}, 5 \mathrm{X}, 6 \mathrm{X}, 7 \mathrm{X}$ and $8 \mathrm{X}$ and above. It was found that age was effective on total $\mathrm{X}$ polysomy and there was a statistical difference between the groups $(\mathrm{p}=0.020)$. It was observed that total $\mathrm{X}$ polysomies increased especially due to increasing age and the highest rate was at the age of 71 and over. This difference is mainly due to the increase of $4 X$ cells found statistically significant between the groups $(p=0.012)$. At the same time, it was observed that the rate of $\mathrm{X}$ monosomies was higher in the early stages (I and II) than in the advanced stage (III and IV) and the difference was statistically significant $(\mathrm{p}=0,020)$.

\section{For statistical analysis:}

Comparisons between groups were applied using The Mann-Whitney Utest and Kruskal-Wallis test were used to compare date that were not normally distributed. The categorical variables between groups were analyzed using the $\chi 2$ test. Results are presented as the mean \pm standard deviation and the median (range). $\mathrm{P}<0.05$ was considered to indicate a statistically significant difference. Statistical analyses were performed using SPSS 11.0 software programme.

\section{Discussion}

Cancer is known to result from a combination of the genetic defects. Also, the tumors display very complex structural CAs the origin of which are often very difficult to estsablish. The identification of recurrent chromosomal changes is a useful strategy for understanding tumorigenesis and specific chromosomal associations. Tumour cells frequently have structural alterations of chromosomes, including deletions, duplications, inversions and translocations. Most frequently, a loss of genetic material in the form of deletions and the other structural CAs also can be observed in BC patients [1]. However, aneuploidy is also features of cancers that are usually associated with poor prognosis. This study investigates the frequency of chromosomal gains and losses in blood and cancer tissues in $\mathrm{BC}$ patients. We reported that numerical sex CAs in cancer tissues were higher than blood tissues and the difference was statistically significant $(\mathrm{p}<0.0001)$. This too indicates that numerical sex chromosomal imbalances found in blood and cancer tissues are often discordant. We can say that these increases in sex chromosome aneuploidies may be due to the characteristic feature of cancer. In a parallel study with our findings, heterogeneous populations of CAs were detected in tumors. In this study; In breast cancer patients 52,04\% (in tumor) and $13,42 \%$ (in lymphocytes), and in healthy blood relatives $9,03 \%$ of metaphases were found aneuploid, and in controls $1.28 \%$. These different values were found statistically high $(\mathrm{P}<0.00001)$. At the same time, in lymphocytes of BC patients, chromosomes $1,6,8,9,15,17,18$, 20 , and $X$ were frequently involved, and gains of $1 \mathrm{q}$ and $8 \mathrm{q}$ were the most common genetic changes. Either one or both of these changes were found in $80 \%$ of unselected $\mathrm{BC}$ [1]. In our study; a lot of damage was observed at chromosome $7 \mathrm{p}-\mathrm{q}, 9 \mathrm{p}, 11 \mathrm{p}-\mathrm{q}, 15 \mathrm{p}, 17 \mathrm{q}$ and $21 \mathrm{q}$ in the lymphocyte cells of $\mathrm{BC}$ patients. It show that it can be inferred from the findings that the above mentioned chromosomes may have an important role in early stage of carcinogenesis in $\mathrm{BC}$ patients, and the autosomal aneuploidies play a role in the pathogenesis of $\mathrm{BC}$, and can be used as indicators of malignancies. Several studies have also shown that anöploidies in different chromosomes are associated with aggressive tumor behavior [4,5]. Chromosomal instability could accelerate the rate of loss of heterozygosity of a tumour suppressor gene and/or effectively amplify an oncogene by duplicating the chromosome on which it lies, thus contributing to tumorigenesis.

In the present study, the incidence of all aneuploidies in patients' blood tissues was about $4 \%$, while only one $\mathrm{X}$ chromosome loss (monosomy) / gain (polysomy) in the examined cancer tissue cells was found to be $36 \%$. And, the frequencies of sex chromosome aneuploidies were higher in the tumoral tissues than in the blood $(\mathrm{p}<0.0001)$. There was a significant difference in the frequencies of SCAs between the tumor and blood tissues, and this was higher in the tumor tissue $(\mathrm{p}<0.0001)$. Our results may suggest that the aneuploidies of $\mathrm{X}$ chromosomes play a role in the pathogenesis of BC. Especially chromosomal gain / loss was associated with poor prognosis, metastasis and tumor progression in breast, ovarian and uterine cervical tumors [6,7]. The X chromosomes have been associated with malignancy in different types of human tumors. Such changes may be important for early cancer detection and may be a diagnostic criterion for breast cancers. At the same time, why sex chromosome increase in cancer tissues are much higher than blood tissues? This shows that it would be more accurate to analyze cancer tissues for the definitive diagnosis, treatment and development of the disease.

$\mathrm{X}$ chromosome aberrations have been found in the neoplasms of different organs. This shows that the presence of numerical aberrations of the $\mathrm{X}$ chromosome indicates that it may play an important role in $\mathrm{BC}$ development. Because, aneuploidy and chromosomal instability are features of cancers that are usually associated with poor prognosis, or a cause or consequence of cancer, and it is known to participate in the progression of the disease. However, sex chromosome aneuploidies may 
therefore offer substantial help in differentiating between non-neoplastic and malignant conditions, may also help to differentiate between benign and malignant lesions. These our observations suggest that the response to aneuploidy might very well be tissue specific. Thus, aneuploidy has a direct effect on the prognosis of patients suffering from breast cancer, and regardless of other parameters we can say that it can be an indicator of poor prognosis.

However, we were also observed that the rate of X monosomia was higher in the early stages of cancer (I and II) than in the advanced stages (III and IV) and the difference was statistically significant $(p=0.020)$. This result shows that aneuploidy is significantly high in the early stages of cancer, and genetic damage is a trigger cause at the beginning of cancer. A distinction between neoplasia and non-neoplastic conditions is based on the presence or absence of clonal chromosome damage. Whereas nonneoplastic lesions were thought to have a normal karyotype, the finding of a clonal abnormality would signify neoplasia. However, it was reported in findings that are not compatible with this. For example, clonal chromosome changes

were found to be significantly different in normal oral mucosa samples in head and neck cancer patients and healthy individuals [8]. However, these clonal CAs in non-neoplastic tissues have been suggested as exemplary exceptions. Why karyotypes may represent cell clone(s) of the tumor has been extensively discussed in the literatures. A most likely explanation is that the malignant cell clones have a growth advantage. It is also possible that some of the abnormal karyotypes may be due to mitoses of malignant cells, because such aberrations are often found in malignant breast lesions.

The loss and gain of the $\mathrm{X}$ chromosome in women with aging is much more frequent than that of the $\mathrm{Y}$ chromosome in males. However, paradoxically, $\mathrm{X}$ chromosome aneuploidy is rarely seen in the dividing cells of the bone marrow in females [9]. A loss of the Y chromosome is frequently observed in myeloproliferative diseases and myelodysplastic syndromes, and can also be seen in lymphoproliferative disorders such as lymphomas [10]. A loss of the Y chromosome in contrast, is a common secondary change in both cancer cells and in a few leukemias. At the same time, the advanced age ( 71 years and above) was effectived in increasing the total $\mathrm{X}$ polysomia in our patients. These findings show that the significantly higher frequency of aneuploidy seen in older ages increases the genomic instability during the progression of the disease. On the other hand, it has been also reported that sex chromosome anöploidies increase with rised age [11]. However, it is known that gender CAs increase with increasing age in lymphocyte cultures of healthy people. Conversely, other studies have shown that age is not clearly related and the $\mathrm{X}$ chromosome that are lost reappeared after therapy and during clinical remission. Therefore, it supports the hypothesis that the loss of sex chromosome is due to the evolution of a malignant clone. However, polysomy $\mathrm{X}$ was also known to be associated with the tumor stage, with a possible correlation with the degree of tumor.
As a result; the cytogenetics karyotyping and FISH should all be considered to obtain accurate diagnoses of malignancies, especially cancer. This highlights the clinical importance of a combined modality approach for the accurate diagnosis and classifi cation of cancers. It seems that to derive sufficient conclusions for the clinical significance of CAs in $\mathrm{BC}$ and to correlate genetic changes with disease, it is necessary that many more cases be studied and that in each case, as many chromosomes or chromosomal regions as possible be evaluated. Our findings show that blood tissue and cancer tissue should be analyzed together for the exact diagnosis, treatment and development of the disease. If possible, the study of fresh cancer tissue should be preferred.

\section{References}

1. Demirhan O, Tanrıverdi N, Süleymanova D. (2016); Chromosomal Aberrations in Patients with Breast Cancer. Journal of Medicine and Health Research. 1(1):23-29.

2. Taştemir Korkmaz D, Demirhan O, Çetinel N, et al. (2017); Sex chromosome alterations in undifferentiated pleomorphic sarcoma. Int J Mol Biol Open Access. 2(1): 00014.

3. ISCN: International system of human cytogenetic nomenclature, Shaffer LG, Slovak ML, Campbell LJ (eds). (S Karger AG, Basel); 2009.

4. Roy SK, Trividi AH, Bakshi SR, et al. (2001); A study of chromosome aneuploidy in hereditary breast cancer patients and their healthy blood relatives. J Exp Clin Cancer Res. 20(1):103-9.

5. Watters AD, Going JJ, Grigor KM, Bartlett JM. (2002); Progression to detrusor-muscle invasion in bladder carcinoma is associated with polysomy of chromosomes 1 and 8 in recurrent $\mathrm{pTa} / \mathrm{pT} 1$ tumors. Eur J Cancer. 38:1593-1599.

6. Ribal MJ, Alcaraz A, Mengual L et al. (2004); Chromosomal highpolysomies predict tumor progression in $\mathrm{T} 1$ transitional cell carcinoma of the bladder. Eur Urol.45:593-599.

7. Piao Z, Malkhosyan SR. (2002); Frequent loss Xq25 on the inactive $\mathrm{X}$ chromosome in primary breast carcinomas is associated with tumor grade and axillary lymph node metastasis. Gene Chromosome Cancer. 33:262-269.

8. Kersemaekers AM, van de Vijver MJ, Kenter GG, Fleuren GJ. (1999); Genetic alterations during the progression of squamous cell carcinomas of the uterine cervix. Gene Chromosome Cancer. 26: 346-354.

9. Jin C, Jin Y-S, Wennerberg J, Akervall J, Dictor M, Mertens F (2002); Karyotypic heterogeneity and clonal evolution in squamous cell carcinomas of the head and neck. Cancer Genet Cytogenet. 132:85-96

10. Jacobs PA, Maloney V, Cooke R, et al. (2013); Male breast cancer, age and sex chromosome aneuploidy. Br J Cancer. 108:959-963.

11. Riske CB, Morgan R, Ondreyco S, Sandberg AA. (1994); X and Y chromosome loss as sole abnormality in acute non-lymphocytic leukemia (ANLL). Cancer Genet Cytogenet. 72:44-47. 